\title{
Regulation of CNS and motor axon guidance in Drosophila by the receptor
}

\section{tyrosine phosphatase DPTP52F}

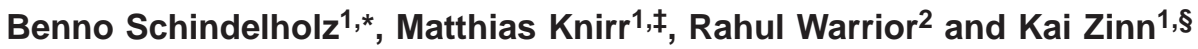 \\ ${ }^{1}$ Division of Biology, California Institute of Technology, Pasadena, CA 91125, USA \\ 2Department of Biology, University of Southern California, 835 W 37th Street, Los Angeles, CA 90089, USA \\ *Present address: The Genetics Company, Winterthurstr. 190, 8057 Zürich, Switzerland \\ fPresent address: Friedrich-Miescher-Labor der Max-Planck-Gesellschaft, Spemannstr. 37, D-72070 Tübingen, Germany \\ §Author for correspondence (e-mail: zinnk@its.caltech.edu) \\ Accepted 10 August 2001
}

\section{SUMMARY}

Receptor-linked protein tyrosine phosphatases (RPTPs) regulate axon guidance and synaptogenesis in Drosophila embryos and larvae. We describe DPTP52F, the sixth RPTP to be discovered in Drosophila. Our genomic analysis indicates that there are likely to be no additional RPTPs encoded in the fly genome. Five of the six Drosophila RPTPs have $C$. elegans counterparts, and three of the six are also orthologous to human RPTP subfamilies. DPTP52F, however, has no clear orthologs in other organisms. The DPTP52F extracellular domain contains five fibronectin type III repeats and it has a single phosphatase domain. DPTP52F is selectively expressed in the CNS of late embryos, as are DPTP10D, DLAR, DPTP69D and DPTP99A. To define developmental roles of DPTP52F, we used RNA interference (RNAi)-induced phenotypes as a guide to identify Ptp52F alleles among a collection of EMSinduced lethal mutations. Ptp52F single mutant embryos have axon guidance phenotypes that affect CNS longitudinal tracts. This phenotype is suppressed in Dlar Ptp52F double mutants, indicating that DPTP52F and DLAR interact competitively in regulating CNS axon guidance decisions. Ptp52F single mutations also cause motor axon phenotypes that selectively affect the $\mathrm{SNa}$ nerve. DPTP52F, DPTP10D and DPTP69D have partially redundant roles in regulation of guidance decisions made by axons within the ISN and ISNb motor nerves.

Key words: Receptor tyrosine phosphatase, PTP, Drosophila, Neural development, Axon guidance, Neuromuscular system, Genomics, Motor axon, RNAi

\section{INTRODUCTION}

Receptor-linked protein tyrosine phosphatases (RPTPs) regulate axon guidance and synaptogenesis decisions in Drosophila embryos and larvae. Five Drosophila RPTPs have been described, and four of these are selectively expressed on central nervous system (CNS) axons (Desai et al., 1994; Hariharan et al., 1991; Streuli et al., 1989; Sun et al., 2000; Tian et al., 1991; Yang et al., 1991). They do not obviously define distinct sets of pathways, as they appear to be expressed on most or all CNS axons. DPTP4E mRNA is widely expressed, but its protein distribution pattern is unknown (Oon et al., 1993).

The four 'neural' RPTPs DPTP10D, DLAR, DPTP69D and DPTP99A have been studied using genetics. All four regulate axon guidance decisions in embryos (Desai et al., 1996; Desai et al., 1997; Krueger et al., 1996; Sun et al., 2000; Sun et al., 2001; Wills et al., 1999). Ptp69D, Dlar (Lar - FlyBase) and Ptp10D mutations also cause optic lobe innervation defects in larvae and pupae (Garrity et al., 1999; Newsome et al., 2000) (T. Suzuki et al., and Q. Sun and K. Z., unpublished).

Single mutants that lack DLAR or DPTP69D have guidance phenotypes affecting specific pathway decisions made by embryonic motor axons (Desai et al., 1996; Krueger et al., 1996). However, most guidance decisions within the neuromuscular system are altered only when specific combinations of two or more RPTPs are eliminated, indicating that RPTPs can have partially redundant functions at growth cone choice points (Desai et al., 1996; Desai et al., 1997; Sun et al., 2000; Sun et al., 2001).

RPTPs can also have 'competitive' activities, in a formal genetic sense. DLAR and DPTP99A function in opposition to each other in regulating entry of the ISNb motor nerve into its target ventrolateral muscle (VLM) field (Desai et al., 1997). The Abl tyrosine kinase and its substrate Ena are also involved in DLAR signaling at this decision point (Wills et al., 1999).

Little is known about the biochemical mechanisms involved in control of motor axon guidance by RPTPs. The ligands and/or co-receptors that might interact with their extracellular (XC) domains have not been identified. The in vivo substrates for fly RPTPs are also unknown, although several proteins that they can dephosphorylate in vitro or in transfected cells have been described (Fashena and Zinn, 1997; Wills et al., 1999).

None of the previously characterized Rptp single mutants has strong phenotypes that affect the array of axons within the CNS, as assayed by staining with several different antibody 
markers. Removal of both DPTP10D and DPTP69D, however, generates a unique phenotype in which many longitudinal axons abnormally cross the midline (Sun et al., 2000). The Ptp10D Ptp69D double mutation interacts with roundabout, commissureless and slit, a set of mutations defining a pathway that repels longitudinal axons from the midline and prevents commissural axons from recrossing it (Zinn and Sun, 1999).

We describe DPTP52F, which is probably the last remaining RPTP encoded in the Drosophila genome. Ptp52F mutations cause specific CNS and motor axon guidance phenotypes, and exhibit genetic interactions with mutations in the other Rptp genes.

\section{MATERIALS AND METHODS}

\section{Genetics}

In an screen to isolate mutations in the Drosophila Lissencephaly 1 (DLis-1; Lis1 - FlyBase) gene, 18,000 independent EMS mutations were isolated (J. Duncan and R. W.; a complete description will be published elsewhere). 183 mutations were identified that are lethal when placed in trans to the deficiency $D f(2 R) J P 6$, which includes the DLis- 1 and Ptp52F genes. These mutations were subdivided by testing for lethality over two overlapping deficiencies, $D f(2 R) J P 4$ and $D f(2 R) J P 8$ (Fig. 1). 70 mutations in 13 complementation groups were lethal in trans to all three deficiencies, and eight Ptp52F alleles were identified among these.

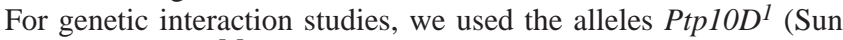
et al., 2000), Dlar 5.5 (Krueger et al., 1996), the transheterozygous combination Ptp69D $/$ IDf(3L)8ex25 (Desai et al., 1996) and Ptp99A ${ }^{1}$ (Hamilton et al., 1995).

\section{Molecular biology}

PCR amplification of DPTP52F, DPTP26C and DPTP36E fragments was performed on phage stocks of a 9-12 hr $\lambda$ gt11 library (Zinn et al., 1988) using primers designed from genomic sequences. cDNA clones were isolated from this and other libraries, including a random-primed 9-12 hour $\lambda$ ZAPII library (K. Z., unpublished), by hybridization to these PCR fragments. We were only able to obtain one cDNA clone encoding a portion of the DPTP52F extracellular (XC) domain. The sequence of this cDNA could encode an open reading frame of 881 amino acids, corresponding to the C-terminal section of the predicted protein CG18243 (1419 amino acids) in Release 2 of GadFly. A number of in-frame stop codons are located upstream of the ATG for the reading frame, which is at nucleotide 580 of our cDNA. However, a comparison of the sequence of our cDNA to the genome sequence revealed that an intron upstream of this ATG had not been completely removed. In the cDNA, the $5^{\prime}$ splice site of this intron is fused to sequences within the intron, and the fusion joins the $5^{\prime}$ splice site to a site near the branchpoint, 14 nucleotides $5^{\prime}$ to the acceptor site. This cDNA is thus a copy of an aberrantly spliced RNA. All other introns within the cDNA sequence were removed in a normal manner. To define the complete coding region for DPTP52F, we then performed reverse transcription-PCR (RT-PCR) experiments on primary embryonic first-strand cDNA preparations. We used a 3' primer within the cDNA, downstream of the aberrantly spliced intron, and a series of $5^{\prime}$ primers, the most $5^{\prime}$ of which was upstream of the ATG of CG18243 (in the presumed 5' UTR of the CG18243 mRNA; primer sequences available on request). (CG18243 has no ESTs, so its predicted mRNA has no 5' UTR; it begins with the ATG of the predicted protein.) Analysis of the cloned PCR products revealed that the DPTP52F protein sequence begins with the initiating methionine of CG18243. There are several in-frame stop codons immediately upstream of this ATG, so the protein must start here. The authentic DPTP52F sequence is very similar to CG18243, except that the intron that was incompletely removed from our cDNA (intron 3 ) does not use the predicted CG18243 5' splice site, but rather uses a $5^{\prime}$ splice site 42 nucleotides downstream. This is the same $5^{\prime}$ splice site that was fused to the branchpoint sequence in our clone. The actual $3^{\prime}$ splice site of this intron is as predicted for CG18243. Joining these splice sites preserves the CG18243 reading frame, so the DPTP52F protein contains an extra 14 amino acids, making the entire preprotein 1433 amino acids in length.

\section{dsRNA-mediated genetic interference}

This was performed essentially as described in the Carthew laboratory protocol (http://www.pitt.edu/ carthew/manual/RNAi_Protocol.html) (Kennerdell and Carthew, 1998; Schmid et al., 2001). Sequences to be transcribed into dsRNAs were cloned into Bluescript $\mathrm{KS}+$, linearized with the appropriate restriction enzymes, and transcribed in vitro with Ambion T3 and T7 Megascript kits following the manufacturer's instructions. Transcripts were annealed in injection buffer $(0.1 \mathrm{mM} \mathrm{NaPO} 4, \mathrm{pH} 7.8 / 5 \mathrm{mM} \mathrm{KCl})$ after heating to $95^{\circ} \mathrm{C}$ for 1 minute and cooled gradually to room temperature by placing the water bath in a foam box over a 18 hour period. We typically injected $\sim 0.1 \mathrm{nl}$ of $0.5-2.0 \mu \mathrm{M}$ solutions of dsRNAs. The Ptp52F dsRNAs corresponded to Ptp52F sequences spanning nucleotides 1279-2444 and nucleotides 2105-3998 (counting from the initiating ATG). Cages were set up using 2- to 4-day-old Oregon R flies. Eggs were collected over a 15- to 30-minute period on grape juice agar plates for subsequent injection. The eggs were lined up lengthwise on double stick tape on a glass slide and immersed in halocarbon oil. Syncitial blastoderm embryos were injected at $\sim 50 \%$ egg length (EL) using an Eppendorf transjector. After injection, embryos were allowed to develop to stage 16-17 under oil in a moist chamber.

\section{Antibody production}

A fragment of the extracellular domain of DPTP52F (amino acids 572-1037) tagged with His6 was expressed in E. coli at the Caltech Protein Expression Facility. Monoclonal antibodies (mAbs) against this fusion protein were generated at the Caltech Monoclonal Antibody Facility and screened based on their ability to recognize the fusion protein on dot blots and by staining of whole mount embryos. $\mathrm{mAb} 13 \mathrm{~B} 8$, which worked well for immunostaining of whole mount embryos (Fig. 2), was used at a 1:3 dilution.

\section{Immunohistochemistry}

Embryos were collected from crosses between flies bearing various combinations of mutant alleles. The desired mutant embryos were identified based on the absence of staining with mAbs specific for each RPTP and/or by the absence of staining with anti- $\beta$-galactosidase $\mathrm{mAb}$ (Promega), which detects the presence of the lacZ gene on the balancer chromosomes. Anti-RPTP mAbs used were 3A6 (DPTP99A) (Tian et al., 1991), 45E10 (DPTP10D) (Tian et al., 1991), 3F11 (DPTP69D) (Desai et al., 1994) and 8C4 (DLAR) (Sun et al., 2000). Mutant embryos were then restained with mAb 1D4 (Van Vactor et al., 1993) to reveal motor axon and CNS pathways, dissected, and photographed with a Magnafire digital camera on a Zeiss Axioplan microscope using Nomarski optics. All mAb supernatants were used at $1: 5$ to $1: 10$ dilutions. Antibody staining using horseradish peroxidase (HRP) immunohistochemistry was performed as described (Patel, 1994). Amplification of the HRP signal was performed using a Vectastain Elite kit (Vector Laboratories).

\section{Database searches}

Celera scaffold sequence (Venter et al., 2001) was searched with blastn using cDNA sequences encoding each human PTP named in a publication. PTP protein sequences were used to search the same database using tblastn, and correlations were made between scaffolds and known RPTPs. Unknown PTP-related sequences were examined further to determine if they are pseudogenes. The names of all 19 human RPTP-like proteins, with an Accession Number for 

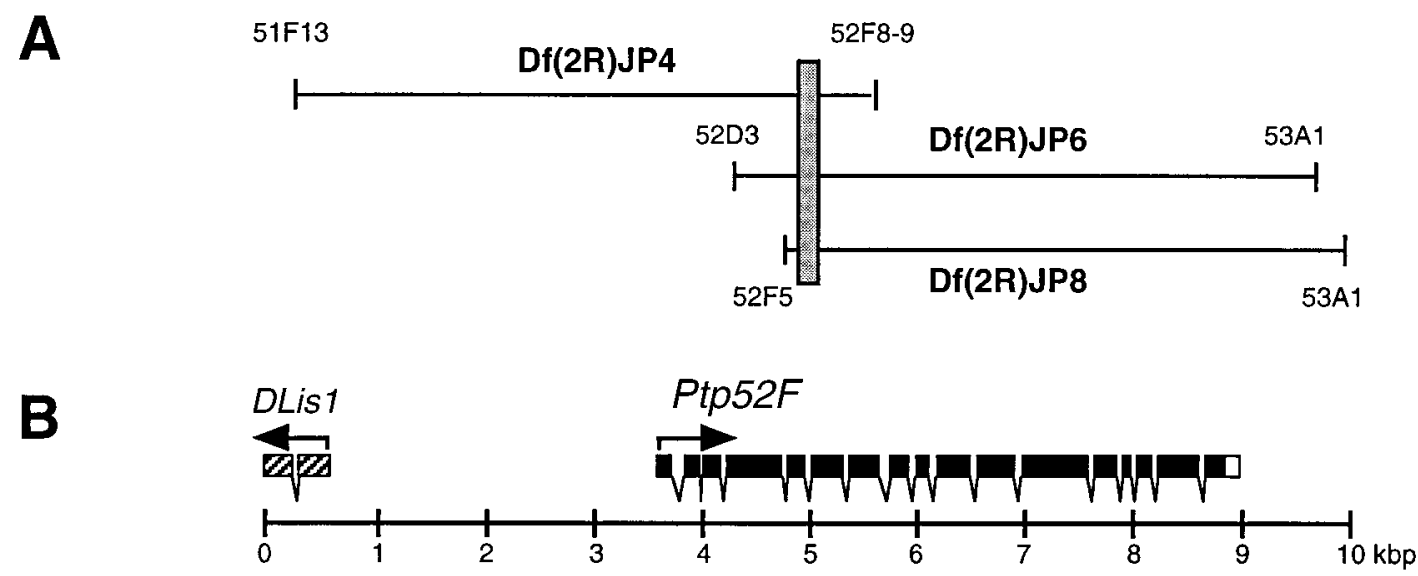

C

1 MEATNWKOSH RRIHALILIL WIAGVOGOTV GSLVAVSESQ ELAEKVEVTV VSTVYTLGFS ISNEADYSID KVICRNGSGS ETEANAAHVC ENLNPCTFYT 101 SVVSFRSNVS GKPPPSDQTI YAYTEYKQPK TTVTSVVATA NTIKVTWQTN DRACVESFGI TAKATDYTKS FQLLNDKSSQ TFVNLSACLT HTITLDTRNN 201 ASVUVDTDAT DVDTQYAEPG DLVMNVTNLA SGITMITWGD PSEKNCISNY VFKWQRNDCG TDQNQDTTTD VPSTETTNEI DYVTITPMET TPDTPSDEVK 301 CEWTDVSSDG KLREYLLTDL QGCDLYTFQV FINENSTAKA SQTFTSAEKL VSAVYEPSPT AYPTQLHWTW FSPQNHPKCV ANYSVTLTGP IQRSENKTMN 401 VITEETFAIF DDLDPCGIYL VEIVPNQLNG SAGTKYQEQS TVGEDQPSVI QDPVVEAEAY SMEVSWKTPE YADLCIDGYR LSGWMEDDKL VEVEALSITT 501 QNTTVVDDKN LLACQVYIIQ IIPYTKENLD GQLRQVGVET KAAIVDYTKV KLEMKNAGSE FIDLIAFNAD YNNSCPTIFA LFTCNATTQV RNPYAERYVE 601 GHSKQGFNAS LSPLSPYATY VCKVILYNVA GRSEPVDDAD MHTTTYYFPEQ PESVMLEKST VSSLLFNWQP PTYTNGPIKY YQAFLMRHEA SYFVPADCAI 701 VEQDTKSETK GDPSVNFTGL APAVRYMMQV AAQNDFGMGV YTEPVIGITL PAVSDSVTQL TVLTOGPVNN NAVYEANVTI TWKVPCKSNG DIEYFQLAFN 801 GTRNNFAPVS FERRVELDTG NKQGRMSYTE TEMQPQFDYT VEVSVKNRDV EQLSSSVPGS WQSPAGLPTI PSDELIKQMR ANVEETSNPT KTAIVRLPAD 901 IMTSASGDIK WMALMISOKN CAGVPHLKYD VSSDWPKVLS YQEAGADGTG DCSLEYQTTE ERWHPEPVOR QRRDGEVTSD EEIVFTIGLD KCSEVQKTYC 1001 NGPLLPDTDY NVVRLFTAS GYSDAAVLNF KTKAAIKVTL ILVSVCSCLL LAFVLGLTVL WVRKRLAWKR DSGQGIEDPF GNVIAKNFAI FYTEVAKPEK 1101 LAREFKEITV VALELSYSAS ELGCHKNRYA DIFPYDKNRV ILDIDAEGSD YINASFIDGH TRKKEYIATQ GPKPESVMDF WRMILQYNVR VIVQVTQERE 1201 GNTIKCHEYY PYNVRGLTVT IKSKEVLELY DRTELTVVHD KYGLKEKVIH YYFKKWPDHG VPEDPMHLIM FVKKVRAEKR PSYSPIVVHC SAGVGRTGTF 1301 IGLDLIMQRL KSESKINIFE TVKKLRFQRM KMVQTQQQYT FLYACTKELV KHKIPRAALK MDGRPKSVTV PAIPSPKKVS FPDVDVGSEY VSSAPITDLD 1401 DGRPIVQLPS RFSGLRRNSP PGENDNPTSS SNM

\section{D}

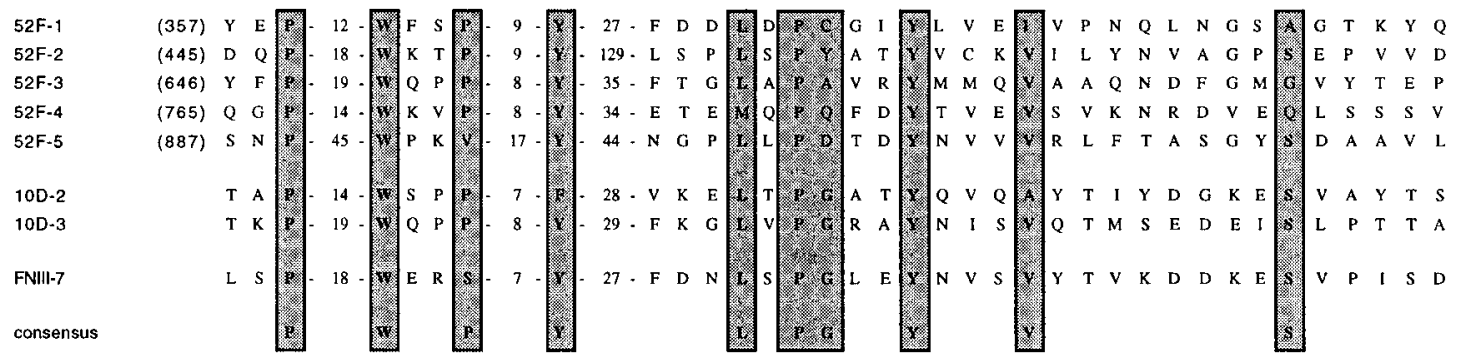

Fig. 1. The Ptp52F gene. (A) Map of the deficiencies used for the EMS lethal screen. The position of the Ptp52F gene is indicated by the shaded bar. (B) Exon maps of Ptp52F and the adjacent DLisl genes; distal (toward the telomere) is towards the left, so the orientation is flipped relative to A. (C) Sequence of the DPTP52F preprotein. Indicated sequences are: putative signal sequence, thin underline; FN3 repeats, thick underlines; putative transmembrane domain, double underline; PTP domain, broken underline; bold, highly conserved PTP domain residues; letters in shaded bars, amino acid changes produced by mutations. (D) Lineup of the DPTP52F FN3 repeats with two FN3 repeats from DPTP10D and the seventh FN3 repeat in human fibronectin. Shaded bars, conserved residues. Consensus residues indicated in bold. 
Fig. 2. Expression of DPTP52F protein. Wholemount embryos were stained with $\mathrm{mAb} 13 \mathrm{~B} 8$ using HRP immunohistochemistry. (A) A ventral view of a gastrulating embryo, showing DPTP52F at the edges of the ventral furrow (arrow). At this stage, DPTP52F is on the membranes of all cells in the embryo at lower levels. (B) A side view (anterior towards the left) of a stage 15 embryo. The ventral nerve cord (arrow) expresses DPTP52F. (C) Two segments of a dissected CNS from a stage 15 wild-type embryo. DPTP52F is expressed on cell bodies; axon tracts (arrow) are lighter. (D) A dissected CNS from a stage 15 Ptp $52 F^{18.3}$ embryo. No staining is observed.

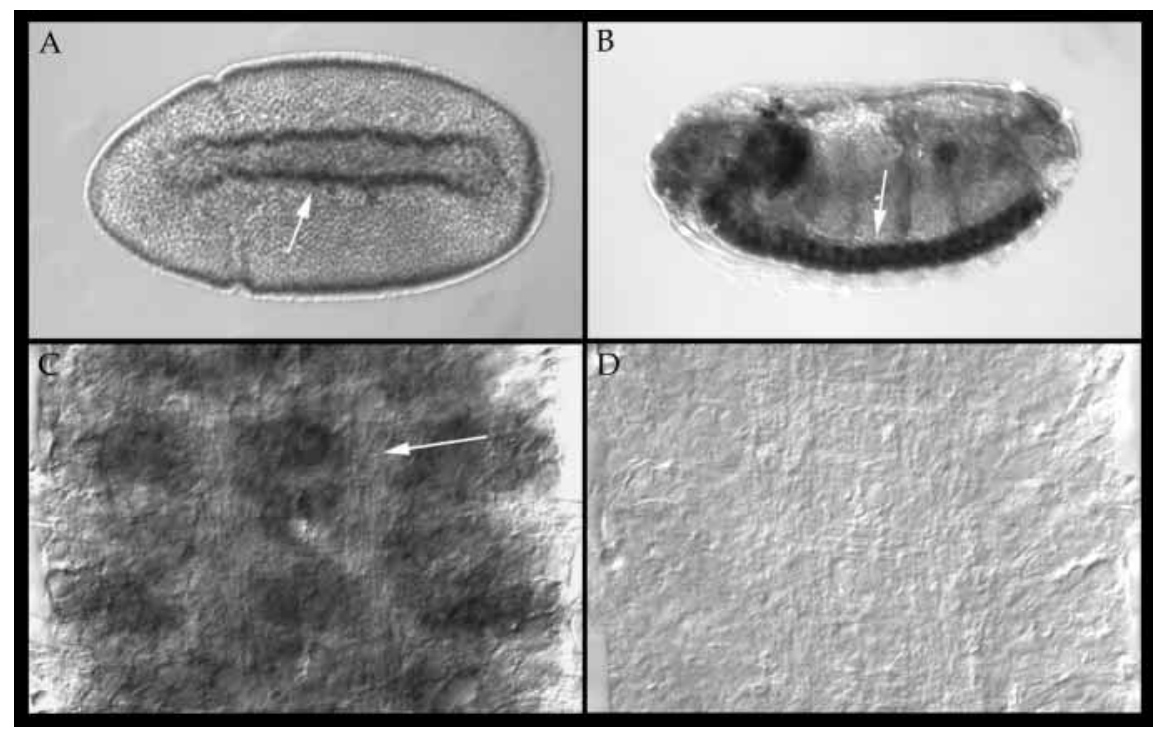

a reference sequence for each, are HPTP $\beta=$ PTP $\beta$ (X54131), HPTP $\eta=D E P-1=C D 148$ (D37781), GLEPP-1=PTP-U2 (U20489), Sap-1 (D15049), PTP-RQ=PTP-GMC1 (human fragment (134 amino acids) is AF169351, rat rPTP-GMC1 (full sequence) is

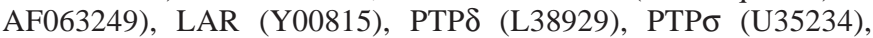
PTP $\mu$ (X58288), PTPк (L77886), RPTP $\rho=$ PTPRT (AF043644), $\mathrm{PTPo}=\mathrm{PTPRO}=\mathrm{hPTP}-\mathrm{J}=\mathrm{PCP}-2=\mathrm{R}-\mathrm{PTP}-\psi=\mathrm{PTP}-\pi,=\mathrm{PTPRU}=\mathrm{PTP} \rho$ (U71075), PTP $\zeta=$ RPTP $\beta$ (M93426), PTP $\gamma$ (L09247), PTP $\alpha$ (M34668), PTPE (X54134), CD45=LCA (Y00062), IA-2=512 antigen=PTP35 (L18983), and phogrin=IA-2 $\beta=P T P-N P=P T P-$ $\mathrm{IAR}=\mathrm{ICAAR}=\mathrm{PTP}-\mathrm{X}(\mathrm{AB} 002385)$ (the last two are catalytically inactive). The reference sequences and scaffolds of the 16 cytoplasmic PTPs and the nine new PTP pseudogenes we defined are available on request. Our conclusions regarding orthologous relationships between fly, worm and human RPTPs differ from those in a recent review (Walchli et al., 2000). These authors defined orthologs based solely on E values of blastp searches with PTP domains, and did not consider whether homology extends over the entire protein. As these $\mathrm{E}$ values are very similar for many pairs of PTP domains, and such values are highly sensitive to gap length, this is not a good way to determine orthologs. For example, they assigned DPTP69D as the CD45 ortholog, and DPTP99A as the PTP $\gamma$ ortholog, but there are no relationships between the XC domain sequences within these pairs.

\section{RESULTS}

\section{Identification and characterization of the sixth Drosophila RPTP gene}

The five previously known Drosophila RPTP genes were all identified by low-stringency hybridization and PCR experiments (Hariharan et al., 1991; Streuli et al., 1989; Tian et al., 1991; Yang et al., 1991; Oon et al., 1993). Three cytoplasmic PTP genes have also been characterized: $c s w$, Ptp$E R$ and Ptp61F (Karim and Rubin, 1999; McLaughlin and Dixon, 1993; Perkins et al., 1992). Mutant alleles have been isolated for all of the Rptp and cytoplasmic PTP genes except Ptp4E and Ptp61F.

We performed searches of the Berkeley Drosophila Genome Project (BDGP) genomic sequence database for additional PTP sequences in 1998 and 1999. When these searches were conducted, about $25 \%$ of the sequence was available, but much of it was in unordered pieces. We found PTP sequence fragments at three cytological locations: $26 \mathrm{C}, 36 \mathrm{E}$ and $52 \mathrm{~F}$. These fragments all encoded a sequence closely related to VHCSAGRGV, the canonical PTP active-site motif (Fauman and Saper, 1996). We isolated and sequenced cDNA clones encoding these three PTPs. Analysis of these sequences showed that we had identified two cytoplasmic PTPs (DPTP26C and DPTP36E) and an RPTP (DPTP52F).

Our analysis of cDNA clones and PCR products showed that the DPTP52F preprotein is 1433 amino acids in length (see Materials and Methods for details). The amino acid sequence immediately following the initiating methionine has a 23 amino acid uncharged region (amino acids 15-38) and resembles a signal peptide. A single predicted transmembrane domain is located at residues 1038-1062. The XC domain has 17 potential N-linked glycosylation sites. Its $\mathrm{C}$-terminal region (amino acids 370-1037) contains five fibronectin type III (FN3) repeats like those found in other Drosophila RPTPs. FN3 repeats are also found in many adhesion and extracellular matrix molecules (Fig. 1D). The N-terminal 370 amino acids of the XC domain is not significantly related to any other sequences in the database.

Among published RPTP sequences, DPTP52F is most similar overall to DPTP4E (42\% similarity), although they differ in the number of FN3 repeats in their XC domains (five versus 13). DPTP52F has a single cytoplasmic phosphatase domain that is $32-36 \%$ identical in sequence to the PTP domains of the other known Drosophila RPTPs.

We were unable to detect DPTP52F mRNA by in situ hybridization to embryos, suggesting that it is expressed at very low levels. We generated mAbs against the XC domain of DPTP52F, and used these to perform immunohistochemical staining of whole-mount embryos. Expression of DPTP52F is observed at the cellular blastoderm stage. During formation of the ventral furrow, DPTP52F can be detected on cells flanking the invaginating region (Fig. 2A).

Later in development (stages 13-17), DPTP52F is selectively expressed in the CNS (Fig. 2B). This CNS specificity resembles that seen for the four 'neural RPTPs' DPTP10D, DLAR, DPTP69D and DPTP99A (Desai et al., 1994; Hariharan et al., 1991; Sun et al., 2000; Tian et al., 1991; Yang 


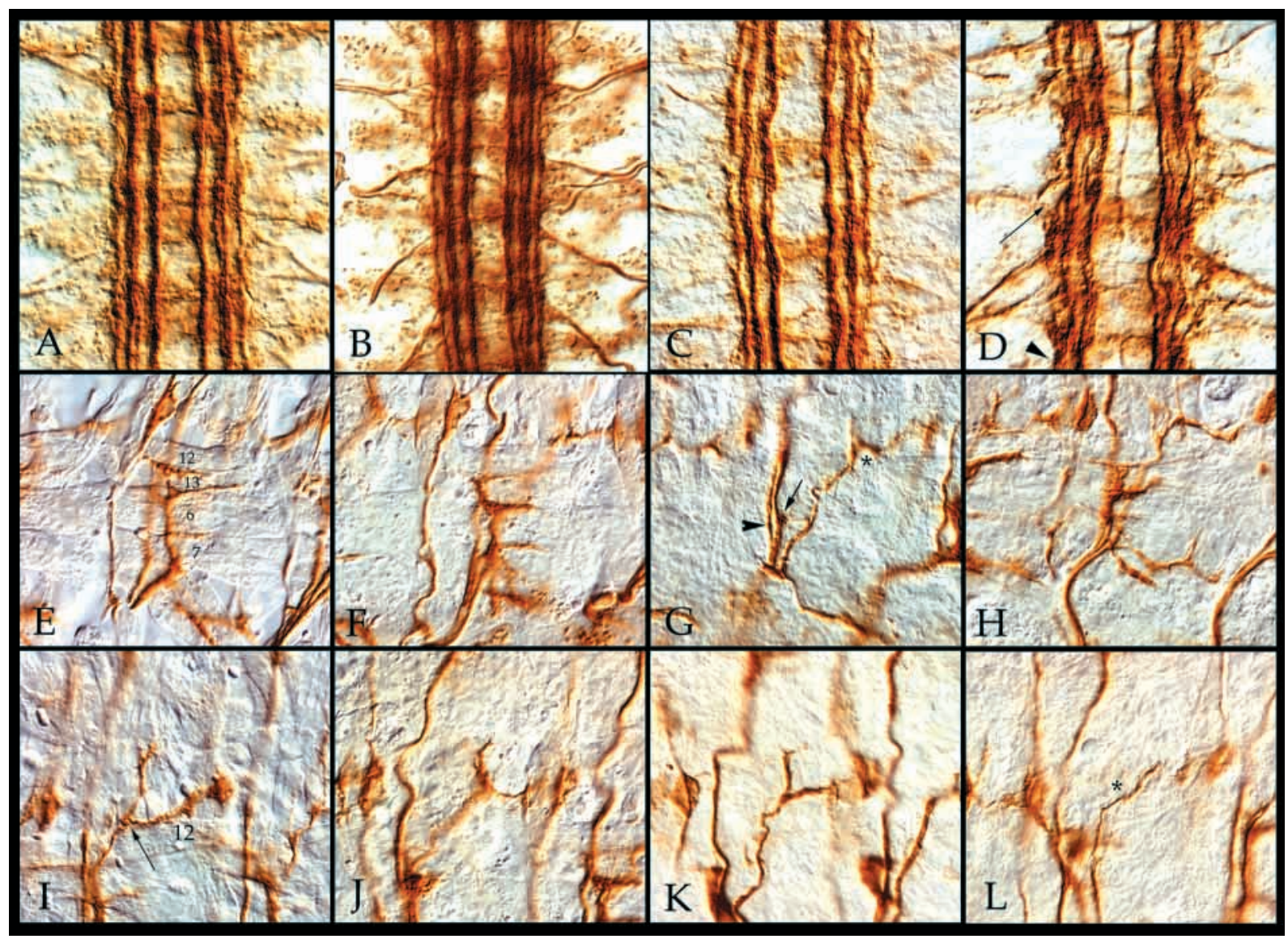

Fig. 3. CNS and motor axon phenotypes produced by RNAi. Dissected late stage 16 embryos stained with mAb 1D4. Column 1 (A,E,I), wildtype. Column 2 (B,F,J), embryos injected with injection buffer. Column 3 (C,G,K), embryos injected with Dlar dsRNA. Column 4 (D,H,L), embryos injected with Ptp52F dsRNA. Row 1 (A-D), the CNS (anterior up); note the 3 straight longitudinal bundles on each side of the midline in A-C. Arrow in D indicates a break in the outer 1D4 bundle; arrowhead indicates abnormal fasciculation of the middle and outer bundles. Row 2 (E-H), ISNb (anterior towards the left); note the 3 characteristic synaptic branches in E,F,H; muscles are numbered in E. Arrow in G, bypass ISNb; arrowhead, ISN; asterisk, SNa. Row 3 (I-L), SNa; note the characteristic bifurcation in I-K, indicated by arrow in (I); muscle 12 is also labeled. Asterisk in L, approximate position of missing bifurcation.

et al., 1991). These RPTPs, however, are localized to axons and are present only at very low levels on neuronal cell bodies. By contrast, DPTP52F appears to be expressed primarily on neuronal cell bodies (Fig. 2C).

By amplifying the antibody signal, we can sometimes detect CNS axon bundles that appear to be positive for DPTP52F staining (data not shown). We cannot state with certainty that these axons express DPTP52F, however, as they are on top of positive cell bodies, and some or all of the brown color of the axon bundles could be due to these underlying cells.

The absence of motor axon staining with anti-DPTP52F is not surprising, because these axon bundles are thin and therefore do not stain strongly. We also cannot visualize expression of DLAR or DPTP99A on motor axons in wild-type embryos, and DPTP10D can only be detected on the SNa nerve (Sun et al., 2000; Sun et al., 2001). Determining that these other RPTPs are axonally localized was not a problem, however, because visualization of positive axon bundles within the CNS was not impaired by staining of underlying cell bodies.

$D f(2 R) J P 4 / D f(2 R) J P 8$ embryos, which have a complete deletion of the Ptp52F gene (Fig. 1A), do not stain with anti-
DPTP52F mAbs. Embryos homozygous for point mutations in Ptp52F (see below) also do not stain (Fig. 2D). These results show that the mAbs selectively recognize DPTP52F.

\section{Perturbation of DPTP52F expression using RNAi produces axon guidance phenotypes}

Injected double-stranded RNA (dsRNA) is a potent and specific inhibitor of gene expression in Drosophila; this method of gene expression blockage is called RNA interference (RNAi) (Kennerdell and Carthew, 1998; Misquitta and Paterson, 1999); see also (Schmid et al., 2001). We initially used RNAi to evaluate the functions of DPTP52F during embryonic development. We examined motor axon and CNS phenotypes in these embryos by staining them with mAb 1D4 (Van Vactor et al., 1993), which recognizes motor axons in the periphery and three paired longitudinal bundles within the CNS. In abdominal segments A2-A7, 32 motor axons exit the CNS within the ISN (intersegmental nerve) and SN (segmental nerve) roots; these then split into five pathways that innervate 30 muscle fibers (Keshishian et al., 1996). The $\mathrm{SNa}$ and $\mathrm{SNc}$ pathways emerge from the SN root, and the ISN, ISNb, and ISNd pathways arise 
from the ISN root. ISNb and ISNd are also known as $\mathrm{SNb}$ and $\mathrm{SNd}$.

To evaluate the efficacy of RNAi for perturbation of Rptp gene expression, we first injected Dlar dsRNA into embryos and allowed them to develop to stage 16-17. Injection of buffer produced no phenotypes (Fig. 3B,F,J), whereas injection of Dlar dsRNA phenocopied the characteristic Dlar mutant ISNb phenotype, known as parallel bypass (Fig. 3G). In parallel bypass hemisegments, ISNb leaves the ISN tract in an apparently normal manner and continues to extend dorsally, but fails to innervate its VLM targets (Krueger et al., 1996). In Dlar RNAi embryos, $15 \%$ of hemisegments had parallel bypass phenotypes. This penetrance is very similar to that observed for zygotic Dlar null mutants (18\%) (Desai et al., 1997). SNa and CNS pathways are not affected in Dlar mutants (Krueger et al., 1996), and these also appeared normal in Dlar dsRNA-injected embryos (Fig. 3C,K).

We then injected two different Ptp52F dsRNAs derived from overlapping sections of the Ptp52F cDNA. The two dsRNAs produced identical phenotypes in which the 1D4-positive bundles in the CNS are irregular and abnormally fasciculated. The outer 1D4-positive bundle is often missing (Fig. 3D).

The ISNb, ISNd and SNc motor nerves appeared identical to wild type in Ptp52F RNAi embryos (Fig. 3H; data not shown). The SNa nerve, however, displayed a clear and diagnostic phenotype. SNa has a characteristic bifurcated morphology. The bifurcation occurs at a choice point located between muscles 22 and 23. The posterior (or lateral) branch of the $\mathrm{SNa}$ innervates muscles 5 and 8, and the anterior (or dorsal) branch innervates muscles 21-24 (Fig. 3I). In embryos injected with Ptp52F dsRNA, either the posterior or anterior branch was sometimes missing (Fig. 3L). The penetrance of this bifurcation phenotype was $18 \%$. In summary, our RNAi experiments suggested that DPTP52F is involved in guidance of longitudinal axons within the CNS and in bifurcation of the SNa motor nerve.

\section{Identification of Ptp52F mutants}

We examined a collection of EMS-induced mutations that are lethal over $D f(2 R) J P 4, D f(2 R) J P 6$ and $D f(2 R) J P 8$ (Fig. 1A) for the Ptp52F CNS and SNa phenotypes that we had defined using RNAi. There are 13 lethal complementation groups in this interval. We collected embryos from lines representing each of these complementation groups and stained them with $\mathrm{mAb}$ 1D4. Mutations within one complementation group produced phenotypes identical to those we had observed with RNAi. This group consists of eight alleles. We sequenced the Ptp52F gene from three of these alleles, and found the following mis-sense mutations (Fig. 1C): Ptp52F ${ }^{18.3}$, Ser to Pro in the fifth FN3 repeat (S941P), Ptp52F 7.8.1, Pro to Ser in the second FN3 repeat (P633S), and Ptp52F $F^{8.10 .3}$, Tyr to Asn in the PTP domain (Y1348N).

Transheterozygous Ptp52F $18.3 / D f(2 R) J P 4$ flies can survive until the first instar larval phase. Ptp $52 F$ embryos appear morphologically normal during all phases of development. The peripheral nervous system (PNS) displays no defects, and the pattern of body wall muscle fibers is also normal. There are also no abnormalities in the patterns of expression of the Evenskipped (Eve) and Engrailed (En) homeodomain proteins during segmentation or later within the CNS, suggesting that the fates of Eve- and En-expressing cells are not altered by these mutations (data not shown).
Table 1. SNa phenotypes in Rptp mutant embryos

\begin{tabular}{|c|c|c|c|c|c|c|}
\hline \multirow[b]{2}{*}{ Genotype } & \multirow[b]{2}{*}{$n$} & \multicolumn{5}{|c|}{ Phenotype $(\%)^{*}$} \\
\hline & & $\mathrm{t}$ & M & $\mathrm{S}$ & A & - \\
\hline \multicolumn{7}{|l|}{ Ptp52F } \\
\hline Ptp $52 F^{18.3}$ & 145 & 37 & 19 & 5 & 14 & 0 \\
\hline Ptp52F $18.3 / D f(2 R) J P 4$ & 137 & 41 & 20 & 7 & 10 & 4 \\
\hline Ptp52F $18.3 / D f(2 R) J P 8$ & 149 & 32 & 9 & 3 & 19 & 0 \\
\hline Ptp $52 F^{8.10 .3^{\circ}}$ & 150 & 28 & 9 & 0 & 18 & 0 \\
\hline$P t p 52 F^{8.10 .3} / D f(2 R) J P 4$ & 100 & 36 & 12 & 4 & 20 & 0 \\
\hline Ptp52F $8.10 .3 / D f(2 R) J P 8$ & 109 & 22 & 7 & 0 & 16 & 0 \\
\hline Ptp52F 7.8 .1 & 138 & 27 & 14 & 0 & 27 & 0 \\
\hline$P t p 52 F^{7.8 .1} / D f(2 R) J P 4$ & 101 & 36 & 12 & 2 & 22 & 0 \\
\hline$P \operatorname{tp} 52 F^{7.8 .1} / D f(2 R) J P 8$ & 129 & 22 & 7 & 0 & 16 & 0 \\
\hline \multicolumn{7}{|l|}{ Ptp10D Ptp52F } \\
\hline Ptp10D Ptp $52 F^{18.3}$ & 175 & 61 & 25 & 33 & 2 & 3 \\
\hline Ptp10D Ptp52F 8.10 .3 & 100 & 45 & 17 & 23 & 5 & 0 \\
\hline \multicolumn{7}{|l|}{ Dlar Ptp52F } \\
\hline Dlar ${ }^{5.5} \operatorname{Ptp}^{2} 5 F^{18.3}$ & 150 & 51 & 22 & 25 & 5 & 0 \\
\hline \multicolumn{7}{|l|}{ Ptp52F Ptp69D } \\
\hline Ptp52F 18.3 Ptp $69 D^{l} / D f(3 L) 8$ ex 25 & 155 & 68 & 36 & 27 & 1 & 4 \\
\hline Ptp52F 8.10 .3 Ptp69D $/ D f(3 L) 8 \operatorname{ex} 25$ & 101 & 51 & 29 & 17 & 2 & 3 \\
\hline \multicolumn{7}{|l|}{ Ptp52F Ptp99A } \\
\hline 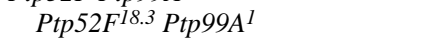 & 145 & 33 & 25 & 3 & 2 & 2 \\
\hline
\end{tabular}

$n$, number of hemisegments (A2-A7) scored.

a, additional branches at random positions; $\mathrm{M}$, either posterior or anterior $\mathrm{SNa}$ branch is missing; $\mathrm{S}, \mathrm{SNa}$ stalls near the bifurcation point; $\mathrm{t}$, all affected $\mathrm{SNa}$ branches; -, the whole SNa branch is missing.

The numbers listed are \% of hemisegments displaying a particular phenotype. Single Dlar, Ptp10D, Ptp69D and Ptp99A mutants are not listed, as these seldom display SNa phenotypes ( $<5 \%$ abnormal hemisegments).

Embryos homozygous for any of the three Ptp $52 \mathrm{~F}$ alleles express no detectable DPTP52F protein, as assayed by staining with anti-DPTP52F mAbs (Fig. 2D). Embryos homozygous for $P \operatorname{tp} 52 F^{18.3}$ or transheterozygous for $P t p 52 F^{18.3}$ over $D f(2 R) J P 4$ have approximately the same phenotypic penetrances. Thus, $P t p 52 F^{18.3}$ may be a null allele. Ptp $52 F^{7.8 .1}$ or $P$ tp $52 F^{8.10 .3}$ produce the same phenotypes as $P \operatorname{tp} 52 F^{18.3}$, but with somewhat lower penetrances, suggesting that they may be hypomorphic alleles (see Table 1, Table 2, Table 3 ). All of these data indicate that the phenotypes described in more detail below are due to the absence of the DPTP52F protein.

\section{Ptp52F mutations perturb guidance of longitudinal pioneer axons}

At stages 16 and 17, mAb 1D4 stains three distinct axon bundles in each longitudinal connective of the CNS, but does not stain any commissural bundles. In Ptp52F $F^{18.3}$ and Ptp52F $18.3 / D f(2 R) J P 4$ embryos, a phenotype identical to that observed in Ptp52F dsRNA-injected embryos is observed, in which the longitudinal 1D4-positive bundles are wavy and irregular and the outer bundle is discontinuous or missing (Fig. 4B,C). The other two alleles produce weaker but similar CNS phenotypes (data not shown). We do not observe clear phenotypes in Ptp52F embryos stained with mAb BP102, which stains all CNS axons (Seeger et al., 1993). This suggests that only a subset of longitudinal axons is affected by the mutations.

To examine the establishment of longitudinal pathways, we analyzed earlier (stage 12 and 13) embryos. During stage 12, $\mathrm{mAb} 1 \mathrm{D} 4$ selectively stains the longitudinal pioneer neurons 
Table 2. ISNb phenotypes in Rptp mutant embryos

\begin{tabular}{|c|c|c|c|c|}
\hline \multirow[b]{2}{*}{ Genotype } & \multirow[b]{2}{*}{$n$} & \multicolumn{3}{|c|}{ Phenotype $(\%)^{*}$} \\
\hline & & $\mathrm{t}$ & B & $\mathrm{S}$ \\
\hline \multicolumn{5}{|l|}{ Ptp52F } \\
\hline Ptp $52 F^{18.3}$ & 145 & 7 & 1 & 6 \\
\hline$P t p 52 F^{18.3} / D f(2 R) J P 4$ & 136 & 13 & 2 & 11 \\
\hline Ptp $52 F^{18.3} / D f(2 R) J P 8$ & 150 & 5 & 1 & 4 \\
\hline Ptp $52 F^{8.10 .3}$ & 150 & 1 & 0 & 1 \\
\hline Ptp52F $F^{8.10 .3} / D f(2 R) J P 4$ & 100 & 4 & 0 & 4 \\
\hline$P t p 52 F^{8.10 .3} / D f(2 R) J P 8$ & 110 & 1 & 0 & 1 \\
\hline Ptp52F 7.8 .1 & 138 & 3 & 0 & 3 \\
\hline Ptp52F $7.8 .1 / D f(2 R) J P 4$ & 100 & 5 & 0 & 5 \\
\hline Ptp52F $7.8 .1 / D f(2 R) J P 8$ & 130 & 2 & 0 & 2 \\
\hline \multicolumn{5}{|l|}{ Ptp10D Ptp $52 F$} \\
\hline Ptp10D 1 Ptp52F $F^{18.3}$ & 175 & 40 & 1 & 39 \\
\hline Ptp10D $10 t p 52 F^{8.10 .3}$ & 100 & 32 & 2 & 30 \\
\hline \multicolumn{5}{|l|}{ Dlar and Dlar Ptp $52 F$} \\
\hline Dlar $^{5.5} /$ Dlar $^{13.2}$ & 260 & 35 & 31 & 4 \\
\hline Dlar ${ }^{5.5}{\text { Ptp } 52 F^{18.3}}^{18}$ & 146 & 40 & 23 & 17 \\
\hline \multicolumn{5}{|l|}{ Ptp69D and Ptp52F Ptp69D } \\
\hline Ptp69D'/Df(3L)8ex 25 & 141 & 3 & 2 & 1 \\
\hline Ptp52F $F^{18.3}{\text { Ptp } 69 D^{1} / D f(3 L) 8 e x 25}$ & 155 & 29 & 3 & 26 \\
\hline Ptp52F $F^{8.10 .3}$ Ptp69D $1 / D f(3 L) 8 \operatorname{ex} 25$ & 101 & 22 & 2 & 20 \\
\hline \multicolumn{5}{|l|}{ Ptp52F Ptp $99 A$} \\
\hline Ptp52F $F^{18.3}$ Ptp $99 A^{1}$ & 145 & 15 & 1 & 14 \\
\hline \multicolumn{5}{|c|}{$n$, number of hemisegments (A2-A7) scored. } \\
\hline \multicolumn{5}{|c|}{$\mathrm{B}$, bypass ISNbs; $\mathrm{S}$, ISNbs that stall within the VLM field; $\mathrm{t}$, all affected } \\
\hline \multicolumn{5}{|l|}{ ISNb branches. } \\
\hline \multicolumn{5}{|c|}{$\begin{array}{l}\text { The numbers listed are } \% \text { of hemisegments displaying a particular } \\
\text { phenotype. Single Ptp } 10 D \text { and Ptp } 99 A \text { mutants are not listed, as these have } \\
\text { no ISNb phenotypes ( }<2 \% \text { abnormal hemisegments). }\end{array}$} \\
\hline
\end{tabular}

MP1, dMP2 and pCC. pCC pioneers the pCC/vMP2 pathway, which extends anteriorly over a 1D4-positive longitudinal glial cell, LG5 (Fig. 5A) (Seeger et al., 1993). When we examined Ptp $52 F$ mutants at slightly later stages (so as to be sure that pCC should have contacted LG5), we observed that the pCC growth cone was usually stalled and had not grown onto LG5 (Fig. 5B).

The stalled pCC phenotype probably represents a delay in growth cone extension rather than a complete block. By stage 13 , the ascending $\mathrm{pCC}$ growth cone encounters the descending axons of dMP2 and MP1. pCC/vMP2 and MP1/dMP2 then establish a continuous longitudinal pathway (Fig. 5C). In Ptp $52 \mathrm{~F}$ embryos examined at a later stage than the wild-type embryo of Fig. 5C, this longitudinal pathway is still thin in $\sim 50 \%$ of hemisegments, and the connection between $\mathrm{pCC}$ and the pathway appears to be missing in some hemisegments (Fig. 5D). This suggests that $\mathrm{pCC}$ growth cones might have retracted or taken an alternate pathway after failing to contact MP1 at the appropriate stage. By stage 15, however, the medial 1D4-positive bundle, which contains the pCC axon (Hidalgo and Brand, 1997), has a relatively normal appearance (Fig. 4B,C).

\section{SNa pathways are altered in Ptp52F and double mutants}

Ptp52F mutants display a variety of SNa guidance defects. The most common defect, as in Ptp52F RNAi embryos, is a failure to bifurcate (Fig. 6C). In other hemisegments, the SNa has extra branches (Fig. 6B), or stalls near the bifurcation point (Fig. 6D). The penetrances of such $\mathrm{SNa}$ phenotypes in Ptp52F18.3
Table 3. ISN phenotypes in Rptp mutant embryos

\begin{tabular}{|c|c|c|c|c|c|c|}
\hline \multirow[b]{2}{*}{ Genotype } & \multirow[b]{2}{*}{$n$} & \multicolumn{5}{|c|}{ Phenotype $(\%)^{*}$} \\
\hline & & $\mathrm{t}$ & $\mathrm{T}$ & SB & FB & $1-$ \\
\hline \multicolumn{7}{|l|}{ Ptp52F } \\
\hline Ptp52F 18.3 & 166 & 16 & 1 & 12 & 2 & 0 \\
\hline$P \operatorname{tp} 52 F^{18.3 / D f(2 R) J P 4}$ & 157 & 20 & 0 & 17 & 3 & 0 \\
\hline Ptp52F $18.3 / D f(2 R) J P 8$ & 175 & 11 & 7 & 2 & 2 & 0 \\
\hline Ptp52F 8.10 .3 & 177 & 5 & 0 & 5 & 0 & 0 \\
\hline$P t p 52 F^{8.10 .3} / D f(2 R) J P 4$ & 120 & 14 & 10 & 4 & 0 & 0 \\
\hline Ptp52F $8.10 .3 / D f(2 R) J P 8$ & 127 & 4 & 4 & 0 & 0 & 0 \\
\hline Ptp52F 7.8 .1 & 160 & 7 & 6 & 1 & 0 & 0 \\
\hline$P t p 52 F^{7.8 .1} / D f(2 R) J P 4$ & 117 & 15 & 6 & 9 & 0 & 0 \\
\hline$P t p 52 F^{7.8 .1} / D f(2 R) J P 8$ & 151 & 5 & 5 & 1 & 0 & 0 \\
\hline \multicolumn{7}{|l|}{ Ptp10D Ptp52F } \\
\hline Ptp10D ${ }^{1} \operatorname{Ptp} 52 F^{18.3}$ & 207 & 67 & 40 & 23 & 4 & 0 \\
\hline Ptp10D 1 Ptp52F 8.10 .3 & 119 & 49 & 35 & 13 & 1 & 0 \\
\hline \multicolumn{7}{|l|}{ Dlar and Ptp52F } \\
\hline Dlar $5.5 /$ Dlar $^{13.2}$ & 256 & 41 & 22 & 19 & 0 & 0 \\
\hline Dlar ${ }^{5.5} \operatorname{Ptp} 52 F^{18.3}$ & 166 & 64 & 51 & 10 & 2 & 2 \\
\hline \multicolumn{7}{|l|}{ Ptp69D and Ptp52F Ptp69D } \\
\hline$P \operatorname{tp} 69 D^{l} / D f(3 L) 8 \operatorname{ex} 25$ & 131 & 1 & 1 & 0 & 0 & 0 \\
\hline Ptp52F 18.3 Ptp $69 D^{1 / D f}(3 L) 8 \operatorname{ex} 25$ & 182 & 58 & 1 & 57 & 1 & 0 \\
\hline Ptp52F $F^{8.10 .3}$ Ptp $69 D^{1} / D f(3 L) 8 \operatorname{ex} 25$ & 117 & 47 & 7 & 40 & 0 & 0 \\
\hline \multicolumn{7}{|l|}{ Ptp52F Ptp99A } \\
\hline Ptp $52 F^{18.3}$ Ptp $99 A$ & 173 & 27 & 4 & 18 & 3 & 1 \\
\hline
\end{tabular}

$n$, number of hemisegments (A2-A7) scored.

FB, ISNs that terminate at the first branchpoint; SB, ISNs that terminate at the second branchpoint; $t$, all affected ISN branches; T, ISNs that appear to reach the terminal arbor position, but are thin or bifurcated; $1-$, ISNs that terminate proximal to the first branchpoint.

The numbers listed are \% of hemisegments displaying a particular phenotype. Single Ptp10D and Ptp99A mutants are not listed, as these have no ISNb phenotypes ( $<2 \%$ abnormal hemisegments).

homozygotes or $\operatorname{Ptp} 52 F^{18.3} / D f(2 R) J P 4$ transheterozygotes are $37 \%$ and $41 \%$, respectively. The two other Ptp52F alleles and the transheterozygous combinations of the three Ptp $52 F$ alleles with $D f(2 R) J P 8$ have a lower penetrance of SNa defects (2228\%; Table 1).

Single mutants that lack any of the other four neural RPTPs do not display SNa phenotypes. However, combinations of Rptp mutations do affect the SNa (Sun et al., 2001). To evaluate how removal of other RPTPs might affect Ptp52F SNa phenotypes, we made double mutants lacking both DPTP52F and each of the other RPTPs. We found that the absence of DPTP10D, DPTP69D or DLAR increases the penetrance of the Ptp $52 F^{18.3}$ defects, particularly those in which the SNa stalls near the bifurcation point. No new phenotypes are observed in double mutants, however. Removal of DPTP99A does not affect the overall penetrance of $\mathrm{SNa}$ phenotypes, but does decrease the frequency of ectopic branches (Table 1).

\section{Ptp10D Ptp52F and Ptp52F Ptp69D embryos display synergistic ISNb phenotypes}

The ISNb motor nerve innervates the VLMs and contains the axons of the identified RP1, RP3, RP4 and RP5 motoneurons. RP growth cones leave the common ISN pathway at the exit junction, enter the VLM field, and then navigate among the muscle fibers. Synapses begin to form at stereotyped positions by late stage 16 (Fig. 7A). Ptp52F mutations produce any detectable ISNb phenotypes only at low frequencies $(<13 \%$; Table 2). 
Fig. 4. CNS phenotypes in Ptp52F and double mutants. Dissected late stage 16 embryos stained with $\mathrm{mAb}$ 1D4. (A) wild-type. (B) Ptp52F 18.3 (C) $P t p 52 F^{18.3} / D f(2 R) J P 4$. The bundles are wavy and disorganized in $\mathrm{B}, \mathrm{C}$, and the outer bundle is sometimes missing (bracket). (B,C,E) Abnormal fasciculation of the middle and outer bundles (arrowheads). (D) Dlar ${ }^{5.5}$ Ptp $52 F^{18.3}$ Bundle morphology in this double mutant reverts to wild type (compare with A-C). (E) Ptp10D ${ }^{1}, P t p 52 F^{18.3}$. (F) Ptp $52 F^{18.3}$,

Ptp69D $^{1 / D f}$ (3L)8ex25. 1D4-positive bundles in these two double mutants are more disorganized than in $\mathrm{B}, \mathrm{C}$, and the middle bundle is strongly affected (asterisks). Arrow, complete connective break.
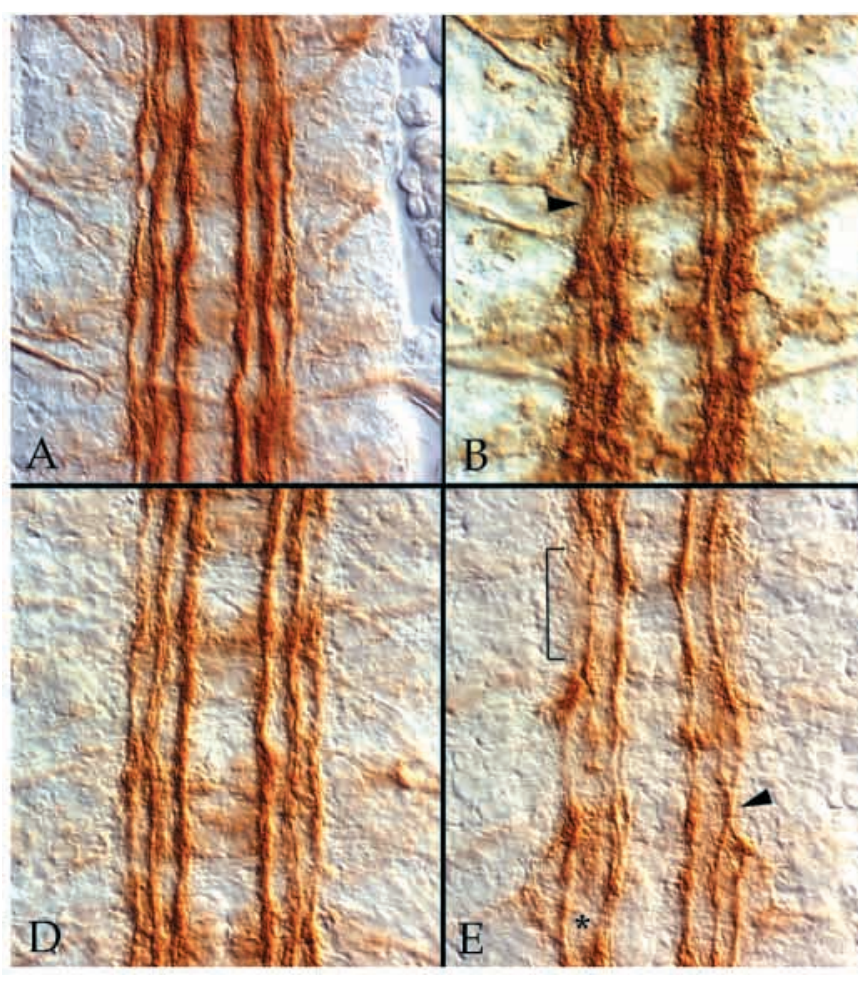

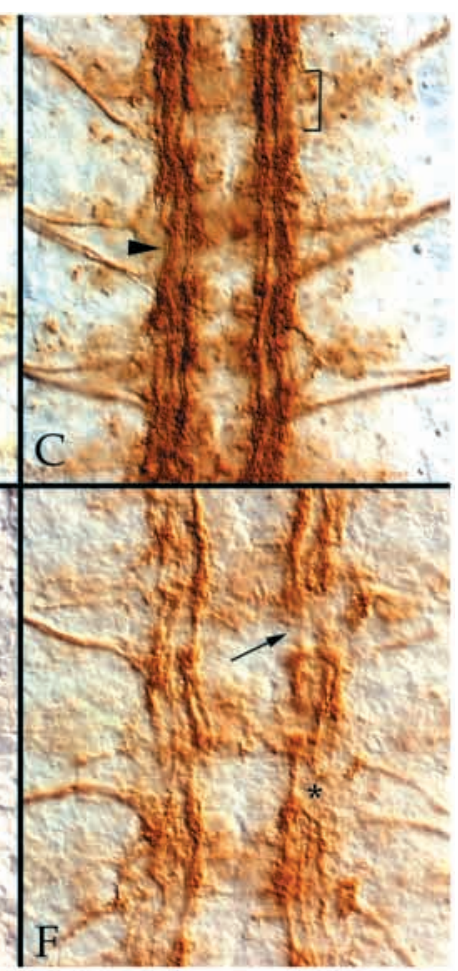

Ptp10D mutations produce no ISNb phenotypes (Sun et al., 2001). Removal of both DPTP10D and DPTP52F, however, generates a strong phenotype in which the ISNb stalls within the VLMs, often at the proximal edge of muscle 13 (Fig. 7B). This stall phenotype is observed in Ptp52F single mutants, but its frequency can be dramatically increased in double mutants $(1 \% \rightarrow 30 \%$ for addition of a PtplOD mutation to the hypomorphic mutation Ptp52F.10.3; Table 2). Removal of DPTP69D also greatly enhances the Ptp52F stall phenotype $\left(1 \% \rightarrow 20 \%\right.$ for Ptp $52 F^{8.10 .3 ;}$ Table 2).

Dlar Ptp52F double mutants have parallel bypass phenotypes identical to those of Dlar single mutants (Krueger et al., 1996). Ptp99A mutations cause no ISNb phenotypes on their own or in combination with Ptp52F (Table 2).

\section{DPTP52F regulates ISN outgrowth}

The ISN passes its first (FB) and second (SB) lateral branchpoints before reaching the position of its terminal arbor at the proximal edge of muscle 1 (Fig. 7C). In Ptp $52 \mathrm{~F}$ mutants, most ISNs are normal (Fig. 7C), but 5-17\% terminate at SB (Table 3). Dlar mutations produce SB phenotypes with a similar penetrance (19\% for null alleles) (Desai et al., 1997). When Dlar and Ptp52F mutations are combined, the frequency of the SB termination phenotype is similar to those of the single
Fig. 5. The pCC growth cone stalls in Ptp52F mutants. Dissected embryos stained with mAb 1D4. (A) wild-type, late stage 12. aCC growth cones (a) have just begun to turn outwards. The pCC growth cone has already grown onto the 1D4-positive LG5 cell (bracket) in the hemisegments on the right (indicated by parallel lines flanking the $\mathrm{pCC}$ axon connection to LG5). (B) Ptp52 $F^{18.3}$, early stage 13 . The aCC growth cone (a) has already turned posteriorly and then begun to extend away from the CNS, but the pCC growth cones (p) have still not reached LG5. (C) Wildtype, early stage 13 . The aCC growth cone (a) is at the same position as the one indicated in $\mathrm{B}$. A continuous longitudinal pathway has formed. pCC axons are indicated by flanking parallel lines. (D) Ptp52F ${ }^{18.3}$, late stage 13 . The aCC axons (a) have already extended beyond the edges of the panel, but there are still gaps in the longitudinal pathways on the left side (arrows) just anterior to the pCC cell bodies.

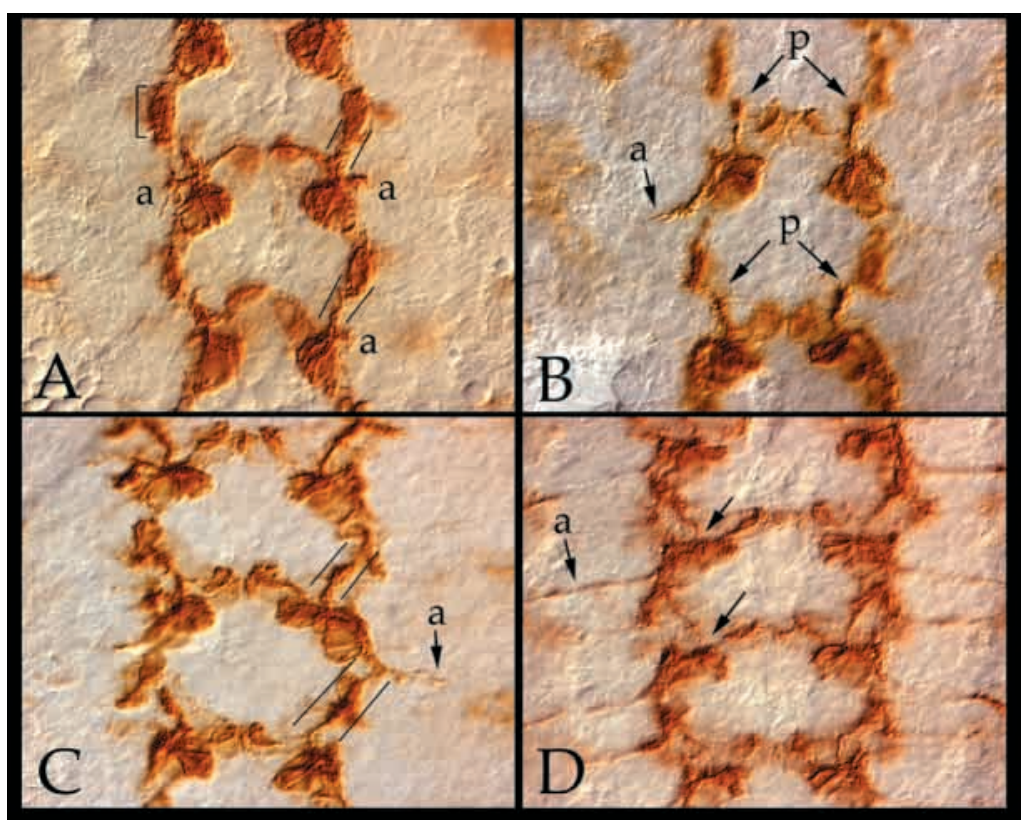




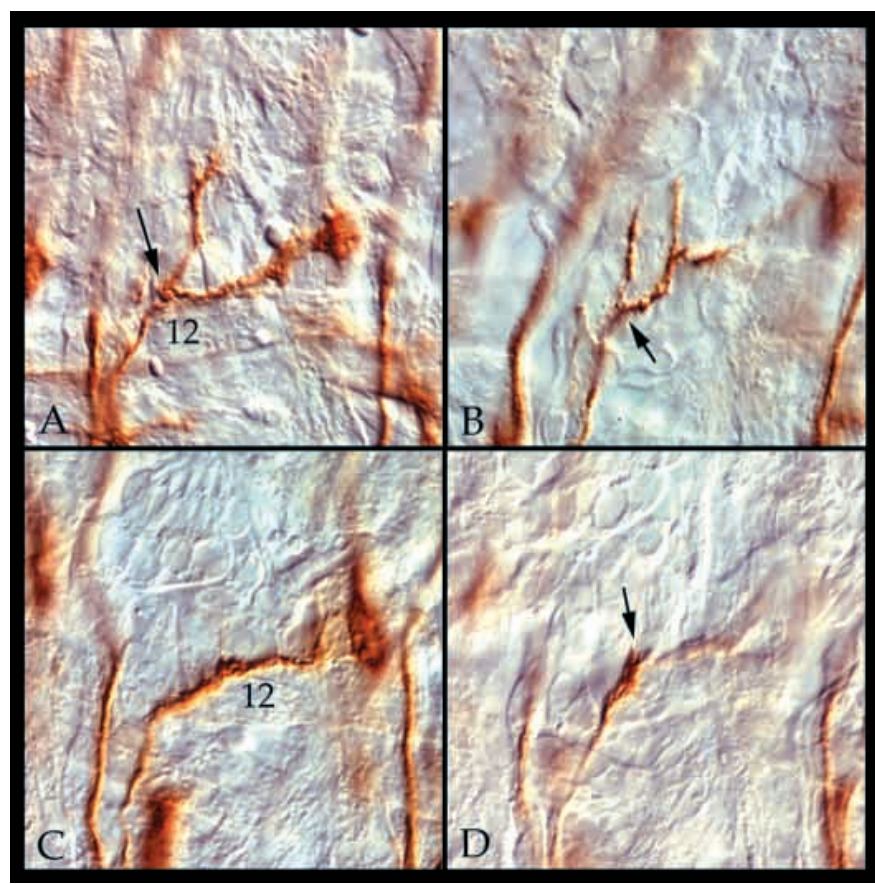

Fig. 6. SNa phenotypes in Ptp52F mutants. Dissected late stage 16 embryos stained with mAb 1D4. (A) Wild type. The SNa bifurcation (arrow) and muscle 12 are indicated. (B-D) Ptp $52 F^{18.3}$. There is an extra branch in B (arrow). The anterior branch is missing in C. Most $\mathrm{SNa}$ axons appear to stall near the bifurcation point in $\mathrm{D}$ (arrow).

mutants. Ptp99A mutations have no effects on ISN on their own, and also cause no enhancement of the Ptp52F phenotype (Table 3).

Ptp10D and Ptp69D single and double mutants have no ISN phenotypes (Fig. 7D) (Sun et al., 2001). However, removal of either of these RPTPs from a Ptp52F mutant background enhances the penetrances of the Ptp52F ISN phenotypes. Ptp10D Ptp $52 F$ double mutants have a reduced terminal arbor (T) phenotype (35\%; Fig. 7E) that is less frequently observed in Ptp52F single mutants $(<10 \%)$. Removal of DPTP69D does not affect the T phenotype, but produces an increase in the SB phenotype $\left(5 \% \rightarrow 40 \%\right.$ for Ptp $52 F^{8.10 .3}$; Table 3; Fig. 7F). In summary, our results indicate that DPTP52F, DPTP10D and DPTP69D have partially redundant functions in regulation of ISN outgrowth. It is interesting that Ptp $52 F$ mutations do not produce synergistic phenotypes when combined with Dlar mutations, which are the only other Rptp mutations that generate strong ISN phenotypes on their own. Perhaps there are two separate 'functions' needed for normal ISN outgrowth, one of which involves DLAR and the other DPTP52F.

\section{Removal of DLAR suppresses the Ptp52F CNS phenotype}

DPTP52F is the only RPTP whose removal produces clear phenotypes in the 1D4-positive longitudinal bundles of the CNS. The 1D4 pathways are usually indistinguishable from wild type in single mutants lacking each of the other four RPTPs. Removal of DPTP10D or DPTP69D from a Ptp52F background strengthens the Ptp52F CNS phenotype. The longitudinal 1D4-positive bundles become more irregular, and frequent breaks and discontinuities in the middle bundle are

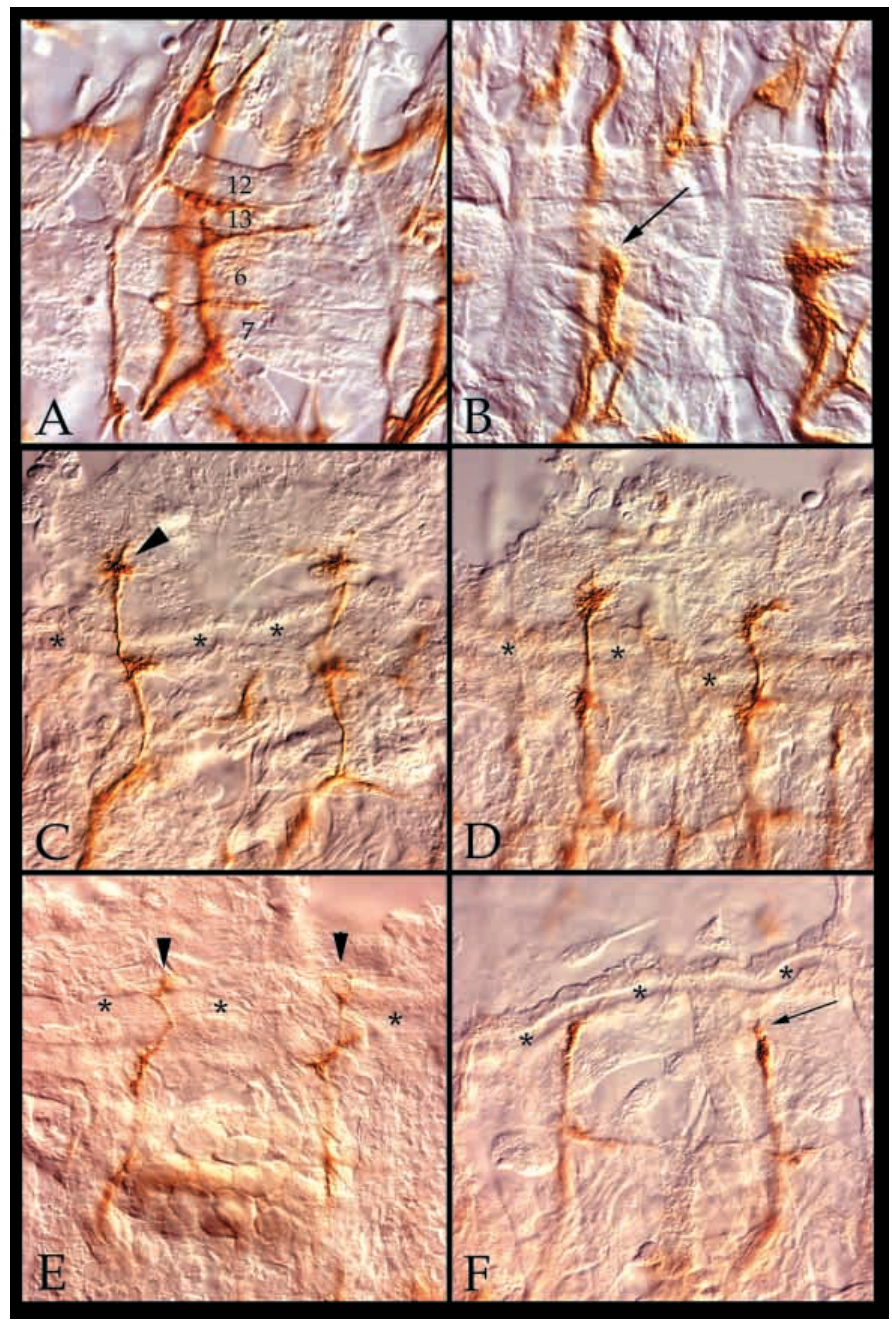

Fig. 7. ISNb and ISN phenotypes in Ptp52F and double mutants. Dissected late stage 16 embryos stained with mAb $1 \mathrm{D} 4$.

(A) Ptp52F ${ }^{18.3}$. The ISNb has a normal appearance. Muscles are labeled. (B) Ptp10D ${ }^{l}, P t p 52 F^{8.10 .3}$. The left ISNb has stalled at the proximal edge of muscle 13 (arrow) without making a synaptic branch. The right ISNb is also truncated. The distal portion of the ISN is shown in C-F. Terminal arbors are indicated by arrowheads in $\mathrm{C}, \mathrm{E}$; the large tracheal branch just distal to the second branchpoint is indicated by asterisks in C-F. (C) Ptp52F $F^{8.10 .3}$.

(D) $\operatorname{Ptp} 69 D^{l} / D f(3 L) 8 e x 25$. The ISN has a normal appearance in these single mutants. (E) $P t p 10 D^{1}$, $P t p 52 F^{8.10 .3}$. Terminal arbors are

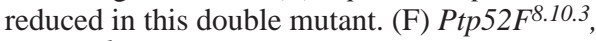

Ptp69D ${ }^{l} / D f(3 L) 8 \operatorname{ex} 25$. ISNs stall at the second branchpoint position (arrow), proximal to the tracheal branch.

observed (Fig. 4E,F). We do not, however, observe a new synergistic phenotype like that produced by removal of DPTP10D and DPTP69D together (Sun et al., 2000). Removal of DPTP99A does not affect the Ptp52F CNS phenotype (data not shown).

In contrast to these results, we find that when a Dlar mutation is introduced into a Ptp52F mutant background, the morphology of the 1D4-positive bundles reverts to wild type (Fig. 4D). In a few segments of Dlar Ptp52F double mutants, breaks in the outer 1D4-positive bundle are still seen, but defasciculation and irregularities in the inner two bundles are not observed. The suppression is specific to the CNS 
phenotypes detected at late stage 16 , because the introduction of Dlar mutations into a Ptp52F mutant background does not correct the failure of the pCC growth cone to extend at the appropriate time.

\section{DISCUSSION}

We describe a new Drosophila RPTP, DPTP52F. This is the sixth RPTP to be identified in the fruit fly, and our analysis indicates that there are unlikely to be any other RPTPs encoded in the genome. DPTP52F is selectively expressed in CNS neurons in late embryos. We isolated mutations in the Ptp52F gene, and showed that Ptp52F mutant embryos have specific motor axon and CNS axon guidance defects. Previous studies by our group and by others have shown that mutations affecting four other RPTPs also produce axon guidance and synaptogenesis phenotypes. In summary, this work suggests that the major functions of at least five of the six Drosophila RPTPs are to facilitate the correct wiring of the nervous system.

\section{RPTPs in flies, worms and humans}

The existence of complete genome sequences for humans, Drosophila and the nematode $C$. elegans allows the determination of how many members of each gene family are required for development and function of these metazoans. We find that Drosophila has 14 genes encoding tyrosine-specific phosphatases: six Rptp genes and eight genes encoding potentially active PTPs without transmembrane domains (cytoplasmic PTPs). The two new cytoplasmic PTPs we identified are now called PEZ/CG9493 (DPTP26C) and CG7180 (DPTP36E). The completed genome sequence allowed identification of three additional PTP genes at $8 \mathrm{~F}$ (CG3101), 61C (CG1228) and 86F (CG14714); none of these is likely to encode RPTPs (Table 4).

These conclusions differ from those in a review of kinase and phosphatase sequences in the fly genome (Morrison et al., 2000), in which CG7180 (DPTP36E) and CG4355 were classified as RPTPs. We isolated and sequenced DPTP36E cDNA clones, and these do not encode an extracellular region. CG4355 is closely related to phogrin, a transmembrane rat phosphatase-related protein expressed on secretory granules, but the Drosophila protein lacks the essential active-site cysteine and has no homology to the extracellular-like (lumenal) domain of phogrin.

C. elegans has many more RPTPs (15) and cytoplasmic PTPs (>57) than does the fly (Table 4) (Hutter et al., 2000; Plowman et al., 1999). To determine how many PTPs are encoded in the human genome, we examined the Celera human genome sequence database (Venter et al., 2001). Surprisingly, our analysis showed that all of the genes that are likely to encode functional human PTPs have already been identified by PCR techniques or by ESTs. The human genome appears to encode 17 active RPTPs and 16 cytoplasmic PTPs. We also found nine PTP pseudogenes that had not been previously identified.

Two human PTP-like genes (IA-2 and phogrin) encode orthologs of CG4355 in Drosophila and ida-1 in C. elegans (Zahn et al., 2001). All of these proteins contain sequence changes at crucial positions, indicating that they do not
Table 4. PTPs in the Drosophila genome

(A) PTP superfamily genes in fly, worm and human

\begin{tabular}{lrcccc} 
Group & Fly & $\begin{array}{c}\text { Fly } \\
\text { fragments }\end{array}$ & Worm* & $\begin{array}{c}\text { Worm } \\
\text { fragments* }\end{array}$ & Human \\
\hline cPTP & 8 & 2 & 57 & 4 & $17^{\ddagger}$ \\
RPTP & 6 & 0 & $26 ?(15)$ & $14 ?$ & 16 \\
DSP & 13 & 4 & 65 & 0 & n.d. \\
LMW & 2 & 0 & n.d. & n.d. & n.d. \\
\hline
\end{tabular}

\section{(B) Complete tyrosine-specific phosphatases}

\begin{tabular}{|c|c|c|c|c|}
\hline GG & Name & Type & Domains & Worm ortholog \\
\hline 6899 & DPTP4E & RPTP & FN3 & F44G4.8 (1e-100) \\
\hline 1817 & DPTP10D & RPTP & FN3 & F44G4.8 (4.4e-104) \\
\hline 10443 & DLAR & RPTP & FN3, Ig & C09D8.1 (0.0) \\
\hline 18243 & DPTP52F & RPTP & FN3 & - \\
\hline 10975 & DPTP69D & RPTP & FN3, Ig & $\begin{array}{l}\text { CLR-1 (1.1e-101) } \\
\text { or C09D8.1 (2e-103) }\end{array}$ \\
\hline 2005 & DPTP99A & RPTP & FN3 & K04D7.4 (6.3e-82) \\
\hline 9181 & DPTP61F & сPTP & & - \\
\hline 3954 & CSW & сPTP & $\mathrm{SH} 2$ & PTP-2 (1.4e-70) \\
\hline 1228 & PTPMEG & cPTP & PDZ, ezrin & PTP-1 (6.1e-156) \\
\hline 9493 & PEZ & сPTP & Ezrin & C48D5.2A (4e-36) \\
\hline 9856 & PTP-ER & cPTP & & - \\
\hline 3101 & & cPTP & & - \\
\hline 14714 & & cPTP & & - \\
\hline 7180 & & cPTP & & T13H5.1 (1.3e-108) \\
\hline
\end{tabular}

*Data from Plowman et al. (Plowman et al., 1999). Hutter et al. (Hutter et al., 2000), however, find only 15 worm RPTPs, and we concur with this number.

†There are also two inactive PTP-like proteins, and at least nine pseudogenes.

cPTP, cytoplasmic PTP; DSP, dual-specificity phosphatase; FN3, fibronectin type III repeat; Ig, immunoglobulin-like domain; LMW, low molecular weight phosphatase; n.d., not determined; RPTP, receptor-linked PTP; -, no clear ortholog.

function as active phosphatases (Cai et al., 2001; Magistrelli et al., 1996).

Three of the six Drosophila RPTPs have human and $C$. elegans counterparts, as follows:

(1) The DPTP10D/DPTP4E subfamily (Type III) is characterized by a large number of $\mathrm{FN} 3$ repeats $(\geq 8)$ and a single PTP domain. It has a single member in $C$. elegans (F44G4.8), two members in Drosophila (DPTP10D and DPTP4E), and five members in humans (НPTP $\beta$, HРTP $\eta$, GLEPP-1, Sap-1 and PTP-RQ). PTP-RQ is only defined in humans by a 134 amino acid fragment, but our analysis indicates that the complete protein is $>2000$ amino acids and is the ortholog of rat rPTP-GMC1.

(2) The LAR subfamily (Type IIa) has three Ig domains and eight or nine FN3 repeats, and two PTP domains. It has a single member in C. elegans (C09D8.1) and Drosophila (DLAR) and three members in humans (LAR, PTP $\delta$, and PTP $\sigma$ ).

Two other fly RPTPs have counterparts in C. elegans but no obvious human orthologs:

(1) DPTP69D has two Ig domains and three FN3 repeats, and is probably orthologous to C. elegans CLR-1.

(2) DPTP99A corresponds to worm K04D7.4. It has three FN3 repeats and two PTP domains, but the second domain lacks the essential cysteine residue. DPTP69D and DPTP99A have been classified as Type IIa (LAR-like) and Type III 
(DPTP10D-like), respectively, but this may be incorrect, as their domain organizations differ from the other members of these subfamilies.

DPTP52F does not have clear nematode or human orthologs. It is equally related to a number of different PTPs. DPTP52F might be classified as a member of the DPTP10D/DPTP4E (Type III) subfamily, however, as it has an XC domain with FN3 repeats and only one cytoplasmic PTP domain.

The 11 C. elegans RPTPs that are not orthologous to Drosophila or human RPTPs all have a nematode-specific extracellular domain denoted as FE (Hutter et al., 2000). This domain has no close relatives in Drosophila or other sequenced species.

The human genome encodes nine RPTPs in four subfamilies not found in worms or flies: (1) MAM domain RPTPs (Type $\mathrm{IIb}$ ), some of which can function as homophilic adhesion molecules (РTP $\mu$, РТРк, РТР $\rho$ and РTPo); (2) Carbonic anhydrase domain-containing (Type V) RPTPs (PTP $\zeta$ and PTP $\gamma$ ); (3) Type IV RPTPs, which have short glycosylated extracellular domains (PTP $\alpha$ and PTPE); and (4) CD45 (Type I). (Most human RPTPs have several names, and one name was arbitrarily selected here; see Materials and Methods for listings of all names).

\section{Regulation of axon guidance by DPTP52F}

To evaluate the functions of DPTP52F during development, we first used RNAi to inhibit its expression. Ptp52F RNAi embryos have distinctive motor axon and CNS phenotypes (Fig. 3). We used these phenotypes as a tool for analysis of a set of lethal mutations within the interval containing the Ptp52F gene. Mutations in one complementation group produced phenotypes identical to those observed with RNAi. We sequenced the Ptp $52 F$ gene from three mutant lines and found nonconservative missense substitution mutations (Fig. 1). Embryos from all three mutant lines fail to stain with antiDPTP52F mAb, suggesting that the mutant proteins do not fold correctly or are unstable (Fig. 2).

We characterized motor axon phenotypes produced by these mutations. In Ptp52F mutants, the SNa motor nerve fails to bifurcate in a normal manner in about $40 \%$ of hemisegments (Table 1). SNa normally splits into anterior and posterior branches at a choice point just distal to muscle 12. Mutant SNa nerves lack either the anterior or posterior branches with approximately equal frequency. $\mathrm{SNa}$ nerves can also have ectopic branches or stall near the normal bifurcation point (Fig. 6).

To further examine the roles of DPTP52F in regulating motor axon guidance, we made double mutants lacking DPTP52F together with each of the other four neural RPTPs. Our earlier work has defined three modes of genetic interaction among Rptp genes: partial redundancy (a guidance decision can be facilitated by any one of a set of RPTPs), collaboration (expression of two specific RPTPs is required to allow a decision to take place) and competition (loss-of-function mutations in one Rptp gene suppress a phenotype produced by mutations in the other gene). Some of these genetic interactions could be explained by formation of RPTP heteromultimers (Sun et al., 2001).

Ptp52F exhibits both partial redundancy and competition in its interactions with the other Rptp genes. It has strong interactions with Ptp10D, Dlar and Ptp69D, but not with
Ptp99A. Interactions with Ptp10D and Ptp69D are synergistic: both the CNS and motor axon phenotypes are worsened in double mutants. In some cases, there is a dramatic difference between double mutants and either single mutant. For example, the ISNb stall phenotype is almost never observed in either Ptp52F (hypomorph) or Ptp10D mutants (1\% of hemisegments), while $30 \%$ of hemisegments have this phenotype in double mutants (Table 2). These kinds of interactions suggest that some process needed for $\mathrm{ISNb}$ extension through the VLM field can be mediated by either DPTP10D or DPTP52F, and this process only fails when both are missing.

Ptp52F embryos have longitudinal tract phenotypes in which the 1D4-positive bundles are irregular and the outer bundle is often missing. These phenotypes are absent in Dlar Ptp52F double mutants, suggesting that DLAR and DPTP52F interact in a competitive manner to regulate longitudinal tract axon guidance/outgrowth decisions (Fig. 4). Dlar does not suppress the Ptp52F SNa or ISN phenotypes, however, so these two RPTPs do not always interact competitively.

DLAR also participates in another competitive interaction: the Dlar ISNb parallel bypass phenotype is absent in Dlar Ptp99A double mutants (Desai et al., 1997). Here, however, it is a Dlar phenotype that is suppressed by removal of another RPTP, rather than the reverse. Ptp52F mutations do not affect Dlar parallel bypass phenotypes. Determination of the mechanisms that underlie these genetic interactions will require biochemical analysis of DPTP5F and of the signaling pathways in which it participates.

We thank Lakshmi Bugga for performing the RT-PCR analysis of the $5^{\prime}$ end of Ptp52F mRNA, Peter Snow (Caltech Protein Expression Facility) for expression of DPTP52F fusion proteins, Susan Ou (Caltech Monoclonal Antibody Facility) for generation of antiDPTP52F mAbs, J. Duncan and Y. Lei for help in generation and complementation analysis of the EMS alleles, Aloisia Schmid for discussions about Ptp52F phenotypes, neuroblast lineages and RNAi, and other members of the Zinn group for discussions and comments on the manuscript. B. S. was supported by postdoctoral fellowships from Caltech and from the Swiss National Science Foundation. This work was supported by NIH RO1 grant NS28182 to K. Z, and by a basic research grant from the March of Dimes Birth Defects Foundation to R. W.

\section{REFERENCES}

Cai, T., Krause, M. W., Odenwald, W. F., Toyama, R. and Notkins, A. L. (2001). The IA-2 gene family: homologs in Caenorhabditis elegans, Drosophila and zebrafish. Diabetologia 44, 81-88.

Desai, C. J., Popova, E. and Zinn, K. (1994). A Drosophila receptor tyrosine phosphatase expressed in the embryonic cns and larval optic lobes is a member of the set of proteins bearing the HRP carbohydrate epitope. $J$. Neurosci. 14, 7272-7283.

Desai, C. J., Gindhart Jr., J. G., Goldstein, L. S. B. and Zinn, K. (1996). Receptor tyrosine phosphatases are required for motor axon guidance in the Drosophila embryo. Cell 84, 599-609.

Desai, C. J., Krueger, N. X., Saito, H. and Zinn, K. (1997). Competition and cooperation among receptor tyrosine phosphatases control motoneuron growth cone guidance in drosophila. Development 124, 1941-1952.

Fashena, S. J. and Zinn, K. (1997). The transmembrane glycoprotein gp150 is a substrate for the receptor tyrosine phosphatase DPTP10D in Drosophila cells. Mol. Cell. Biol. 17, 6859-6867.

Fauman, E. B. and Saper, M. A. (1996). Structure and function of the protein tyrosine phosphatases. Trends Biochem. Sci. 21, 413-417.

Garrity, P. A., Lee, C. H., Salecker, I., Robertson, H. C., Desai, C. J., Zinn, 
K. and Zipursky, S. L. (1999). Retinal axon target selection in Drosophila is regulated by a receptor protein tyrosine phosphatase. Neuron 22, 707-717.

Hamilton, B. A., Ho, A. and Zinn, K. (1995). Targeted mutagenesis and genetic analysis of a Drosophila receptor-linked protein tyrosine phosphatase gene. Roux's Arch. Dev. Biol. 204, 187-192.

Hariharan, I., Chuang, P.-T. and Rubin, G. M. (1991). Cloning and characterization of a receptor-class phosphotyrosine phosphatase gene expressed on central nervous system axons in Drosophila melanogaster. Proc. Natl. Acad. Sci. USA 88, 11266-11270.

Hidalgo, A. and Brand, A. H. (1997). Targeted neuronal ablation-the role of pioneer neurons in guidance and fasciculation in the CNS of Drosophila. Development 124, 3253-3262.

Hutter, H., Vogel, B. E., Plenefisch, J. D., Norris, C. R., Proenca, R. B., Spieth, J., Guo, C. B., Mastwal, S., Zhu, X. P., Scheel, J. et al. (2000) Cell biology: Conservation and novelty in the evolution of cell adhesion and extracellular matrix genes. Science 287, 989-994.

Karim, F. D. and Rubin, G. M. (1999). PTP-ER, a novel tyrosine phosphatase, functions downstream of Ras1 to downregulate MAP kinase during Drosophila eye development. Mol. Cell 3, 741-750.

Kennerdell, J. R. and Carthew, R. W. (1998). Use of dsRNA-mediated genetic interference to demonstrate that frizzled and frizzled 2 act in the wingless pathway. Cell 95, 1017-1026.

Keshishian, H., Broadie, K., Chiba, A. and Bate, M. (1996). The Drosophila neuromuscular junction: A model for studying development and function. Annu. Rev. Neurosci. 19, 545-575.

Krueger, N. X., Van Vactor, D., Wan, H. I., Gelbart, W. M., Goodman, C. S. and Saito, H. (1996). The transmembrane tyrosine phosphatase DLAR controls motor axon guidance in Drosophila. Cell 84, 611-622.

Magistrelli, G., Toma, S. and Isacchi, A. (1996). Substitution of two variant residues in the protein tyrosine phosphatase-like PTP35/IA-2 sequence reconstitutes catalytic activity. Biochem. Biophys. Res. Commun. 227, 581588

McLaughlin, S. and Dixon, J. E. (1993). Alternative splicing gives rise to a nuclear protein tyrosine phosphatase in Drosophila. J. Biol. Chem. 268, 6839-6842.

Misquitta, L. and Paterson, B. M. (1999). Targeted disruption of gene function in Drosophila by RNA interference (RNA-i): A role for nautilus in embryonic somatic muscle formation Proc. Natl. Acad. Sci. USA 96, 14511456.

Morrison, D. K., Murakami, M. S. and Cleghon, V. (2000). Protein kinases and phosphatases in the Drosophila genome. J. Cell Biol. 150, F57-F62.

Newsome, T. P., Asling, B. and Dickson, B. J. (2000). Analysis of Drosophila photoreceptor axon guidance in eye-specific mosaics. Development 127 851-860.

Oon, S. H., Hong, A., Yang, X. H. and Chia, W. (1993). Alternative splicing in a novel tyrosine phosphatase gene (DPTP4E) of Drosophila-melanogaster generates 2 large receptor-like proteins which differ in their carboxyl termini. J. Biol. Chem. 268, 23964-23971.

Patel, N. H. (1994). Imaging neuronal subsets and other cell types in wholemount Drosophila embryos and larvae using antibody probes. In Drosophila melanogaster: Practical Uses in Cell and Molecular Biology. Vol. 44 (ed. E. Fyrberg and L. S. B. Goldstein), pp. 446-488. San Diego, CA: Academic Press.
Perkins, L. A., Larsen, I. and Perrimon, N. (1992). corkscrew encodes a putative protein tyrosine phosphatase that functions to transduce the terminal signal from the receptor tyrosine kinase torso. Cell 70, 225236.

Plowman, G. D., Sudarsanam, S., Bingham, J., Whyte, D. and Hunter, T. (1999). The protein kinases of Caenorhabditis elegans: A model for signal transduction in multicellular organisms. Proc. Natl. Acad. Sci. USA 96, 13603-13610.

Schmid, A., Schindelholz, B. and Zinn, K. (2001). Combinatorial RNAi: a method for evaluating the functions of gene families in Drosophila. Trends Neurosci. (in press).

Seeger, M., Tear, G., Ferres-Marco, D. and Goodman, C. S. (1993) Mutations affecting growth cone guidance in Drosophila: genes necessary for guidance toward or away from the midline. Neuron 10, 409-426.

Streuli, M., Krueger, N. X., Tsai, A. Y. M. and Saito, H. (1989). A family of receptor-linked protein tyrosine phosphatases in humans and Drosophila. Proc. Natl. Acad. Sci. USA 86, 8698-8702.

Sun, Q., Bahri, S., Schmid, A., Chia, W. and Zinn, K. (2000). Receptor tyrosine phosphatases regulate axon guidance across the midline of the Drosophila embryo. Development 127, 801-812.

Sun, Q., Schindelholz, B., Knirr, M., Schmid, A. and Zinn, K. (2001) Complex genetic interactions among four receptor tyrosine phosphatases regulate axon guidance in Drosophila. Mol. Cell. Neurosci. 17, 274-291.

Tian, S.-S., Tsoulfas, P. and Zinn, K. (1991). Three receptor-linked proteintyrosine phosphatases are selectively expressed on central nervous system axons in the Drosophila embryo. Cell 67, 675-685.

Van Vactor, D., Sink, H., Fambrough, D., Tsoo, R. and Goodman, C. S. (1993). Genes that control neuromuscular specificity in Drosophila. Cell 73, 1137-1153.

Venter, J. C., Adams, M. D., Myers, E. W., Li, P. W., Mural, R. J., Sutton, G. G., Smith, H. O., Yandell, M., Evans, C. A., Holt, R. A. et al. (2001) The sequence of the human genome. Science 291, 1304-1353.

Walchli, S., Colinge, J. and van Huijsduijnen, R. H. (2000). MetaBlasts: tracing protein tyrosine phosphatase gene family roots from Man to Drosophila melanogaster and Caenorhabditis elegans genomes. Gene $\mathbf{2 5 3}$, 137-143.

Wills, Z., Bateman, J., Korey, C. A., Comer, A. and Van Vactor, D. (1999). The tyrosine kinase Abl and its substrate Enabled collaborate with the receptor phosphatase Dlar to control motor axon guidance. Neuron 22, 301312

Yang, X., Seow, K. T., Bahri, S. M., Oon, S. H. and Chia, W. (1991). Two Drosophila receptor-like tyrosine phosphatase genes are expressed in a subset of developing axons and pioneer neurons in the embryonic CNS. Cell 67, 661-673.

Zahn, T. R., Macmorris, M. A., Dong, W. J., Day, R. and Hutton, J. C. (2001). IDA-1, a Caenorhabditis elegans homolog of the diabetic autoantigens IA-2 and phogrin, is expressed in peptidergic neurons in the worm. J. Comp. Neurol. 429, 127-143.

Zinn, K., McAllister, L. and Goodman, C. S. (1988). Sequence analysis and neuronal expression of fasciclin I in grasshopper and Drosophila. Cell 53, 577-587.

Zinn, K. and Sun, Q. (1999). Slit branches out: a secreted protein mediates both attractive and repulsive axon guidance. Cell 97, 1-4. 